\section{„Mit Darbepoetin alfa werden optimale Hämoglobinzielwerte dauerhaft stabil erreicht"“}

Mit der Einführung von rekombinantem humanem Erythropoetin (r-HuEPO) Ende der 1980er-Jahre ist die Behandlung der renalen Anämie bei Patienten mit terminaler Niereninsuffizienz revolutioniert worden. Seit 2001 steht mit Darbepoetin alfa (Aranesp ${ }^{\circledR}$ ) der erste lang wirksame Erythropoese-stimulierende Faktor (ESF) zur Verfügung, der sich gegenüber konventionellem r-HuEPO durch eine höhere biologische Effektivität und durch längere Dosierungsintervalle bis hin zur einmal monatlichen Gabe auszeichnet. Über den Stellenwert lang wirksamer ESF im Anämiemanagement sprach für Dialyse aktuell Marianne E. Tippmann mit Prof. Hermann Haller, Direktor der Abteilung für Innere Medizin/Nephrologie an der Medizinischen Hochschule Hannover.

Die Entschlüsselung des Erythropoetin-Gens vor rund 20 Jahren durch den Amgen-Forscher Dr. Fu-Kuen Lin war der Auftakt für eine revolutionäre Entwicklung in der Anämietherapie, die 2001 schließlich in dem innovativen Erythropoese-stimulierenden Faktor NESP ${ }^{1}$ mündete. Worin unterscheidet sich dieses - besser als Darbepoetin alfa bekannte - Protein strukturell von Epoetin alfa? Welche Veränderungen wurden vorgenommen, um das pharmakologische Profil zu verbessern?

Prof. H. Haller: Die absolute Neuerung bei Darbepoetin alfa besteht darin, dass die Glykosylierung verändert wurde: Durch Austausch von fünf Aminosäuren konnten zwei zusätzliche Kohlenhydratseitenketten in das Molekül eingebaut und damit mehr Bindungsstellen für Sialinsäure geschaffen werden. Mit einem höheren Sialinsäuregehalt nimmt wiederum die Serumhalbwertszeit von Erythropoetin-Isoformen zu.

Dieses insgesamt aufwendige Verfahren wird als sogenanntes Glyco-Engineering bezeichnet und stellt einen völlig neuen Zweig innerhalb der pharmazeutischen Industrie dar. Rekombinant hergestellte Moleküle werden in wesentlichen Eigenschaften so modifiziert, dass ein innovativer Wirkstoff entsteht. Der Weg dahin ist allerdings sehr mühsam: Der Suche nach geeigneten Glykosylierungsstellen, die außerhalb der Rezeptorbindungsregion

${ }^{1} \mathrm{NESP}=$ novel erythropoiesis stimulating protein liegen und dennoch die Halbwertszeit verlängern, folgt eine gezielte Veränderung der Molekülstruktur. Schließlich werden die neuen Moleküle auf ihre Funktionalität überprüft, also Rezeptorbindung und Wirkung auf die Erythropoese. Am Ende dieses komplexen Prozesses stand das Darbepoetin alfa, das angesichts seiner neuen Molekülstruktur mit zwei zusätzlichen Kohlenhydratketten und bis zu 22 Sialinsäureresten über optimierte pharmakokinetische Eigenschaften verfügt.

Was genau bewirkt dieses GlycoEngineering? Oder anders gefragt: Welche Vorteile verbinden sich mit einem hyperglykosylierten Epoetin? lich auch mit dem neuen Erythropoesestimulierenden Protein erreicht hat, ist eine verlängerte Serumhalbwertszeit. Verglichen mit der Vorgängersubstanz weist Darbepoetin alfa aufgrund seines erhöhten Kohlenhydrat- und Sialinsäureanteils eine zwei- bis viermal längere Halbwertszeit und damit eine deutlich höhere biologische Aktivität in vivo auf. Die Serumkonzentration bleibt länger oberhalb des erythropoetischen Schwellenwertes, was in einer effizienten kontinuierlichen Stimulation der Erythropoese resultiert.

Für den anämischen Patienten bedeutet dies nicht nur, dass die Substanz in längeren Intervallen als herkömmliche kurz wirksame Erythropoetine appli-
Haller: Was man angestrebt und letzt-

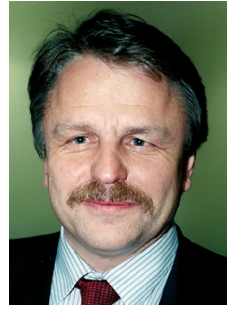

Hermann Haller zierbar ist, sondern man muss auch weniger von ihr geben, um die gleiche Wirkung zu erzielen.

Sind für die biologische Aktivität weitere kinetische Schlüsselparameter maßgeblich? Spielt hier womöglich auch die Affinität für den EPO-Rezeptor eine Rolle?

Haller: Im Unterschied zu traditionellen Arzneistoffen, bei denen klassische pharmakologische Parameter eine Rolle spielen, reden wir hier ja von Substanzen, die direkt mit Molekülen interagieren - in diesem Fall mit dem Erythropoetinrezeptor.

Nun könnte man denken, die höhere Invivo-Bioaktivität von Darbepoetin alfa resultiert aus einer längeren oder auch stärkeren Bindung an den EPO-Rezeptor. Dem ist aber nicht so, und es wäre auch nicht sinnvoll, weil eine starke Rezeptorbindung immer mit der gleichzeitigen Induktion von hemmenden Mechanismen einhergeht. Beispiel r-HuEPO: Hier wird bei starker Rezeptorbindung der ESF-Rezeptorkomplex in die Zelle internalisiert und dort in den Lysosomen abgebaut.

Darbepoetin alfa weist hingegen eine geringere Rezeptoraffinität auf als r-HuEPO, das heißt es bindet kürzer und dissoziiert schneller, sodass es langsamer abgebaut wird. Dadurch kann es zu mehr Rezeptorkontakten kommen: Das Molekül findet sich häufiger zur Signalbildung am Rezeptor ein, aktiviert ihn, geht dann wieder in die Zirkulation. So paradox es klingt, haben wir also trotz verkürzter Bindungszeit eine häufigere Stimulation des Rezeptors als unter konventionellem r-HuEPO, die zu einer höheren biologischen Aktivität in vivo führt.

Dieses neue kinetische Modell, das maßgeblich zum Verständnis des lang wirksamen Erythropoetins Darbepoetin alfa beigetragen hat, ist noch relativ jung, sodass es erst unlängst von den 
Autoren Gross und Lodish zur Publikation kam (3).

Welche klinischen Vorteile verbinden sich mit einem lang wirksamen Erythropoese-stimulierenden Faktor?

Haller: Von klinischer Relevanz sind vor allem drei entscheidende Vorteile: Bedingt durch die verlängerte terminale $\mathrm{Se}$ rumhalbwertszeit kann zum einen der Wirkspiegel im Blutkreislauf länger über der minimalen, die Erythropoese stimulierenden Konzentration gehalten werden als bei äquimolarer Dosis von r-HuEPO. Aus dieser längeren Wirkdauer resultiert wiederum eine verringerte Applikationsfrequenz, das heißt Darbepoetin alfa kann seltener verabreicht werden als ein kurz wirksames Erythropoetin. Und schließlich bewirkt die stärkere Stimulation des EPO-Rezeptors eine stabile Einstellung des Hämoglobinwertes.

Nun sind ja für das Anämiemanagement bei terminaler Niereninsuffizienz bestimmte Zielbereiche für den Hb-Wert vordefiniert. Wie lauten hier die aktuellen Empfehlungen der KDOQI²-Leitlinien?

Haller: Nach den neuen KDOQI-Guidelines vom Mai 2006 liegt die untere Grenze des optimalen therapeutischen Hb-Zielbereichs bei 11,0 g/dl, die Obergrenze bei 13,0 g/dl. Diese Werte werden mit dem besten Überleben der Patienten assoziiert.

Werden diese Zielbereiche mit Darbepoetin alfa dauerhaft erreicht, oder sinkt die In-vivo-Aktivität bei längeren Dosierungsintervallen $a b$, sodass es einer Dosisanpassung bedarf?

Haller: Das Ziel einer stabilen Hb-Einstellung innerhalb der aktuell von der KDOQI definierten Grenzbereiche wird von Darbepoetin alfa dauerhaft erreicht. Eine Dosisanpassung ist also nur dann notwendig, wenn die klinischen Umstände des Patienten, wie zum Beispiel akute Entzündungen oder auch gastrointestinale Blutungen, es erfordern. Da schon ein zeitweises Über- oder Unterschreiten der Hämoglobinzielwerte zu Komplikationen führen kann, liegt es auf der Hand, dass sich mit dieser Stabilisierung auch die Prognose der Patienten deutlich verbessern lässt.

$2 \mathrm{KDOQI}=$ Kidney Disease Outcomes Quality Initiative, National Kidney Foundation
Mit welchen Komplikationen ist ein zu hoher $\mathrm{Hb}$-Wert, mit welchem Outcome ein zu niedriger $\mathrm{Hb}$-Wert assoziiert?

Haller: Die Anpassung der KDOQI-Leitlinien resultiert aus der kritischen Überprüfung der Studienlage zur prognostischen Relevanz von Hämoglobinwerten. Es gibt zahlreiche große Erhebungen, die zeigen, dass das Anheben des Hämoglobinwertes über $11 \mathrm{~g} / \mathrm{dl}$ neben einer Verbesserung der Lebensqualität auch zu einer besseren Prognose der Patienten hinsichtlich Morbidität und Mortalität führt.

Ein zu niedriger Hämoglobinwert beeinträchtigt die Leistungsfähigkeit des Patienten. Der Organismus versucht, dies zu kompensieren, das Herz pumpt mehr, was die Entwicklung einer linksventrikulären Hypertrophie begünstigt. Schon bei einem zeitweisen Unterschreiten des $\mathrm{Hb}$ Zielbereichs, für das natürlich auch weitere Faktoren, wie etwa Eisenverluste, ursächlich sein können, erhöht sich das Mortalitäts- und Hospitalisierungsrisiko für den Patienten.

Was man allerdings auch beobachten konnte, ist eine Art „U-Kurve“ zwischen Hämoglobinwert und Outcome: Geht der Hämoglobinwert zu hoch, kommt es zu Veränderungen im Strömungsverhalten des Blutes und damit zu einer Belastung des vaskulären Systems, die von einer verstärkten Neigung zur Hypertonie bis hin zu Thrombosen reichen kann. In zwei jetzt publizierten Studien ließ sich zudem sehr eindrucksvoll zeigen, dass eine Anhebung des $\mathrm{Hb}$-Spiegels über $13 \mathrm{~g} / \mathrm{dl}$ keinen zusätzlichen Benefit für den Patienten bringt $(2,4)$.

Insgesamt nehmen also die Komplikationen zu, wenn der Hämoglobinwert nicht gut in den Zielbereich von 11-13 g/dl eingestellt ist. Eine dauerhaft stabile Hämoglobineinstellung ist demzufolge als Sicherheitsparameter zu betrachten.

Sie sagten, dass unter Darbepoetin alfa auch bei Verlängerung der Dosierungsintervalle eine gute und dauerhafte $\mathrm{Hb}$-Einstellung erreicht wird. Gibt es klinische Studien, in denen diese Stabilität nach evidenzbasierten Kriterien an dialysepflichtigen, chronisch nierenkranken Patienten nachgewiesen wurde?

Haller: Die Effektivität der Anämietherapie mit Darbepoetin alfa ist bei einer Viel- zahl von Hämodialysepatienten untersucht worden und dementsprechend gut dokumentiert, und zwar in den unterschiedlichsten Applikationsrouten. Inzwischen gibt es zahlreiche prospektive klinische Studien, in denen nachgewiesen werden konnte, dass nach Umstellung von einer zwei- bis dreimal wöchentlichen Gabe eines kurzwirksamen Erythropoese-stimulierenden Faktors auf eine wöchentliche Applikation von Darbepoetin alfa signifikant mehr Patienten den Hämoglobinzielbereich erreichen, unabhängig davon, ob es intravenös oder subkutan verabreicht wurde. Gleiches gilt für die Umstellung von einmal wöchentlich konventionelles r-HuEPO auf einmal 14tägig Darbepoetin alfa.

Und das ist nur eine relevante Erkenntnis aus den Untersuchungen, die für einen lang wirksamen Erythropoese-stimulierenden Faktor spricht: Die Applikationsfrequenz lässt sich deutlich vermindern, was für die Patienten natürlich sehr entlastend ist und damit wesentlich zu einer verbesserten Lebensqualität beiträgt.

Die zweite Frage war: Kommt es denn bei Intervallverlängerung tatsächlich auch zu einer Dosiseinsparung? Und auch hier war das Ergebnis verschiedener Studien eindeutig, die ganz klar zeigten, dass der zur Aufrechterhaltung der Hämoglobinspiegel erforderliche Dosisbedarf für Darbepoetin alfa zum Teil erheblich unter dem äquimolaren Konversionsverhältnis 1:200 lag. Abhängig davon, wieviel Erythropoetin der Patient zur Behandlung seiner renalen Anämie benötigt, reicht die Dosisreduktion von $14-25 \%$ bis hin zu $33 \%$ bei einem wöchentlichen Dosisbedarf von 6000 IE r-HuEPO (1).

Profitieren auch nichtdialysepflichtige Patienten von der unter Darbepoetin alfa reduzierten Applikationsfrequenz?

Haller: Keine Frage - die geschilderten Verbesserungen treffen natürlich auch für nichtdialysepflichtige Patienten zu. Seit die monatliche subkutane Gabe von Darbepoetin alfa zugelassen ist - das ist jetzt gut zwei Jahre her - profitieren die vorher mit einer einmal 14-tägigen Applikation stabil eingestellten Patienten von dem verlängerten Dosierungsregime.

Ist die mittlere wöchentliche Dosierung von Darbepoetin alfa abhängig vom Körpergewicht des Patienten, 
oder kann eine Einheitsdosis verabreicht werden?

Haller: Hier gibt es natürlich Empfehlungen, dass man eine mittlere wöchentliche Dosierung geben kann. Wenn wir aber der Auffassung sind, in die Beurteilung eines Medikamentes und seines therapeutischen Erfolges gehören Kriterien wie beispielsweise Lebensqualität, so hat der Patient auch Anspruch auf eine individuelle Sicht seines Arztes. Ich bin also ganz eindeutig der Meinung, Standardisierung ja, wenn die Behandlung beginnt, und dann muss man sich im Einzelfall je nach Hb-Verlauf den Dosisbedarf der Patienten genau anschauen - der hängt ja nicht allein vom Gewicht, sondern vor allem von Begleiterkrankungen und auch von den akuten klinischen Erfordernissen des Patienten ab. Und auf diese unterschiedlichen Bedürfnisse muss man individuell und flexibel mit der Dosierung, aber auch mit der Applikationsfrequenz reagieren können. So ist beispielsweise eine monatliche Gabe eher für stabile Patienten geeignet, während bei klinisch labileren Patienten die wöchentliche Verabreichung wahrscheinlich mehr Sinn macht.

? Sie erwähnten die erheblichen Dosiseinsparungen unter Darbepoetin alfa, die unter pharmakoökonomischen Gesichtspunkten natürlich eine wesentliche Entlastung bedeuten. Welche vorteilhaften Aspekte - neben verbesserter Lebensqualität - verbinden sich denn mit dem verlängerten Dosierungsintervall - für den chronisch nierenkranken Patienten und auch für den ihn behandelnden Arzt?

Haller: Sicher ist es auch ein wichtiger Aspekt, dass verlängerte Dosierungsintervalle bei stabil eingestellten Patienten zu Entlastungen im Personalbereich führen - obwohl dies natürlich auch von den Organisationsstrukturen des jeweiligen Dialysezentrums abhängt. Auf der anderen Seite möchte ich das Argument der Zeitersparnis nicht als so gewaltig erachten. Wichtig für die Entscheidung, welches Erythropoetin eingesetzt wird, sollten die Kriterien sein, die für den Patienten eine Verbesserung darstellen - und da spricht eben viel für Darbepoetin alfa.

? Warum hat nach Ihrer Einschätzung Darbepoetin alfa noch keine Konkurrenz? Ist der Herstellungsprozess derart komplexer Proteine 'unnachahmlich'?
Haller: Das Wort 'unnachahmlich' trifft genau den Punkt - der Prozess ist das Produkt! Wie schon anfangs kurz angesprochen, liegt ein elementarer Unterschied zwischen chemischen und rekombinant hergestellten Arzneimitteln im Herstellungsverfahren, das für Biopharmazeutika mit extrem aufwendigen Reinigungsund Formulierungsschritten verbunden ist. Dieser hoch komplexe Produktionsprozess muss natürlich eng gesteuert und in all seinen Einzelpassagen streng kontrolliert werden, weil bereits winzigste Änderungen zu dramatisch variierenden und womöglich fatalen Reaktionen im Organismus des Patienten führen können. Und das macht diese sogenannten Biosimilars, deren Hersteller den Anspruch erheben, qualitativ gleichwertige rekombinante Moleküle produzieren zu können, für mich so kritisch.

Zelllinie und Herstellungsverfahren komplexer Moleküle sind ja Eigentum des Innovators und damit einzigartig für dessen Produkt. Abweichungen vom Original und die den Proteinen eigene Komplexität werden demzufolge unweigerlich zur Variabilität bei Nachfolgeversionen führen. Nehmen wir die Änderung der Glykosylierungsstellen beim Darbepoetin alfa als Beispiel: Eine Modifikation im Molekül, die zu veränderten Funktionen führt - in diesem Fall mit sehr positiven Folgen; es kann aber auch völlig gegenteilig laufen.

Bevor also Biosimilars auf den Markt gebracht werden können, müssen die Qualitätsnachweise in der Herstellung erbracht und das lückenlose Beherrschen der Techniken einwandfrei nachgewiesen worden sein. Es ist also noch sehr viel Arbeit notwendig, ehe wir überhaupt eines Tages außerhalb der Lizenz hergestellte Erythropoetine einsetzen können.

Literatur

1 Bock A et al. for the Swiss EFIXNES Investigators. ASN 2004: Poster F-PO518; Abstract MP 204

2 Drüeke TB, Locatelli F, Clyne $\mathrm{N}$ et al. for the CREATE Investigators. Normalization of hemoglobin level in patients with chronic kidney disease and anemia. N Engl J Med 2006 355: 2071-2084

3 Gross AW, Lodish HF. Cellular trafficking and degradation of erythropoietin and novel erythropoiesis stimulating protein (NESP). J Biol Chem 2006; 281 (4): 2024-2032

4 Singh AK, Szczech L, Tang KL et al. for the CHOIR Investigators. Correction of anemia with epoetin alfa in chronic kidney disease N Engl J Med 2006; 355: 2085-2098

Aranesp ${ }^{\circledR}$ ist ein Produkt von Amgen 